\title{
OBITUARIO HANS NIEMEYER FERNÁNDEZ (1921-2005)
}

\section{In memoriam Hans Niemeyer Fernández - Don Hans (1921-2005), testimonio de Andrés Troncoso M. ${ }^{1}$}

Es difícil escribir una reseña en homenaje a Hans Niemeyer Fernández, Don Hans, por varias razones. Primero, porque sin duda alguna es una de las grandes figuras de la arqueología chilena, por lo que es mucho lo que se puede escribir al respecto. Segundo, porque no hay arqueólogo chileno que no lo conozca, por lo que no es tanto lo que se puede escribir sobre él que el resto no lo sepa. Ante este panorama creo que lo mejor es rememorar a Don Hans desde mis recuerdos.

Mis primeros encuentros con Don Hans fueron a partir de la bibliografía, cuando en los años de universidad e interesado en la arqueología del Norte Chico lo conocí por sus ya clásicos trabajos en Puerto Aldea, Huana y otros múltiples sectores del Norte Semiárido, que dejaban traslucir todo un esfuerzo, amor y entusiasmo hacia la arqueología en un tiempo en que, sin duda, en comparación a hoy, era bastante más difícil ser arqueólogo y hacer arqueología.

Tras ese primer encuentro bibliográfico, vino una segunda aparición, en este caso como memoria. Trabajamos en el curso superior del río Illapel en el marco de un proyecto Fondecyt cuando uno de nuestros amigos lugareños nos habla de Don Hans, quien había trabajado con el arte rupestre de este lugar hacía ya unos años. Ahora teníamos un amigo en común: Don Mario Tapia. Desde este momento dos cosas sucedieron. Primero, nació en mí una sospecha que luego se confirmó. Segundo, en cada terreno a Illapel donde veíamos a don Mario Tapia, venía la consiguiente conversación sobre Don Hans y el envío de saludos.

El tercer encuentro fue el definitivo, la corporización de Don Hans. Luego de leer y escuchar de él, tanto por quienes he nombrado como por toda la comunidad arqueológica, surge la posibilidad de ir a excavar a Iglesia Colorada en Copiapó. Fue ese el momento en el que realmente conocí a Don Hans. Uno puede leer muchas cosas de él, entrever otras tantas a partir de tales lecturas, pero creo que no es posible entender el tamaño de su figura sin haberlo conocido. Pienso que aquí de hecho radicará el principal problema de explicarle a las futuras generaciones la significación de Don Hans para la arqueología chilena; podremos contarles muchas cosas, explicar otras tantas y pasarles millones de artículos, pero nada de ello hará justicia a su persona, a su riqueza como ser humano y naturalista. $\mathrm{Su}$ legado traspasa las hojas escritas para poner en primera línea la pasión y el compromiso por la arqueología y el pasado; creo que esa es la lección más grande que él nos dio.

El cuarto encuentro fue el más reciente y ocurrió en un ámbito donde Don Hans fue a todas luces uno de los pioneros en Latinoamérica. Me refiero al estudio del arte rupestre. Sin duda alguna,

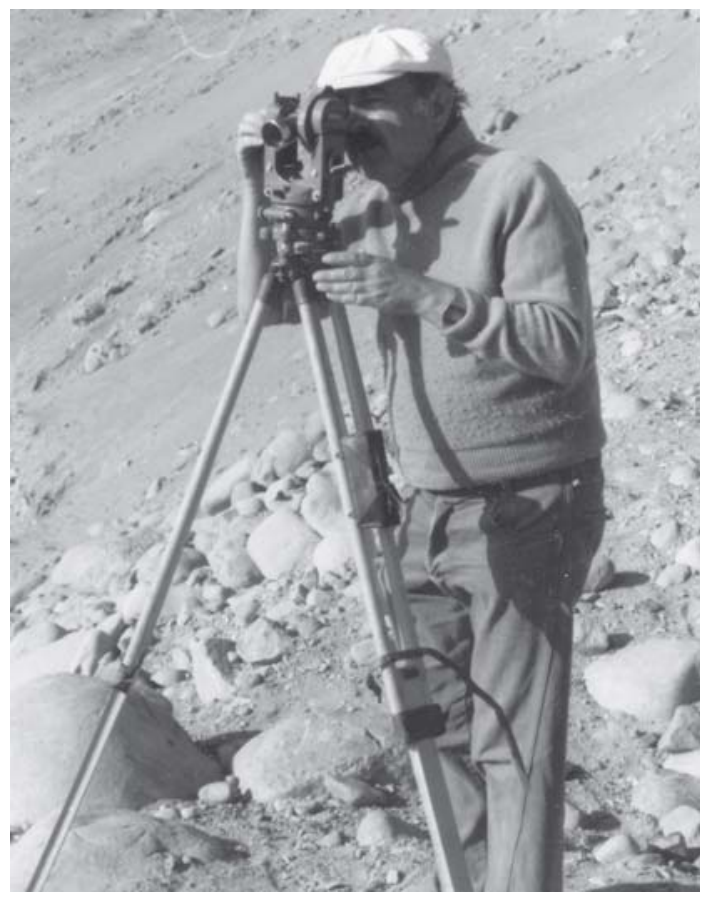

Hans Niemeyer planimetreó innumerables sitios arqueológicos a lo largo de Chile. Enseñó este arte y esta técnica de hacer mapas en las carreras de topografía y arqueología de la Universidad de Chile (Camarones, invierno 1983, fotografía gentileza de C. Santoro). 
cualquier periodización sobre las perspectivas de investigación con relación al arte rupestre en Chile se marca por un momento clave, antes y después de Hans Niemeyer. Fue su trabajo el que impulsó en múltiples zonas del país el estudio de este tipo de materialidad que en muchas ocasiones no era el eje central de las investigaciones arqueológicas. A partir de un detallado relevamiento en terreno y estudio posterior, fue organizando el amplio conjunto de datos que manejaba dentro de lo que es una de las tareas esenciales en arte rupestre, pero también una de las más complejas: su ordenación cronológica cultural. Definió estilos y secuencias, las que, si bien pueden hoy en día y a futuro ser reevaluadas, son, sin duda alguna, la piedra angular para cualquier trabajo sobre el tema, pues en ellos se abren en muchos casos los primeros caminos, que son siempre los más difíciles. La titánica tarea de Don Hans se constituye hoy en día posiblemente en el esfuerzo más amplio y sistemático por abordar este tipo de registro en el país.

$\mathrm{Y}$ creo que es el trabajo en arte rupestre, el que él mismo definió como el trabajo de su vida, donde se puede visualizar de forma clara lo que implicó Don Hans, pues a través de él no sólo dio

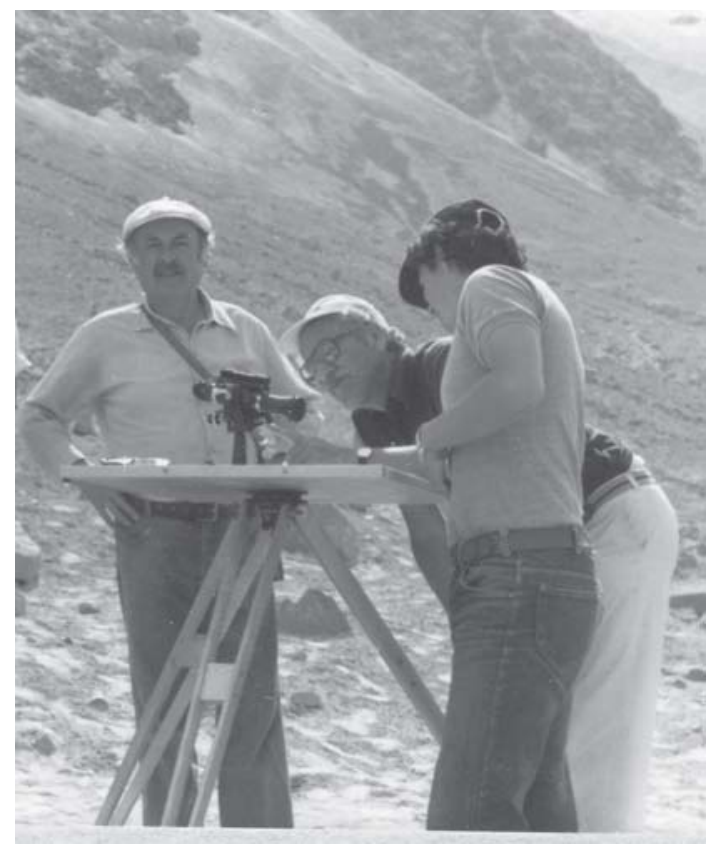

Hans, Iván Solimano y Juan Chacama en el valle de Camarones, tratando de descifrar el uso del instrumento de topografía alidada (invierno de 1983, fotografía gentileza de C. Santoro). cuenta de la variación del registro arqueológico a nivel nacional, y fue capaz de manejar la información dispersa a lo largo del país, comparando y relacionando diseños entre diferentes zonas, sino que trabajó hasta los últimos días de su vida, ya sea en campañas en terreno o en gabinete, a partir de una experiencia humana de la arqueología, siempre acompañado por la señora Selva, ya sea en una terraza fluvial, ya sea en una cima de cerro. Don Hans siempre tuvo la

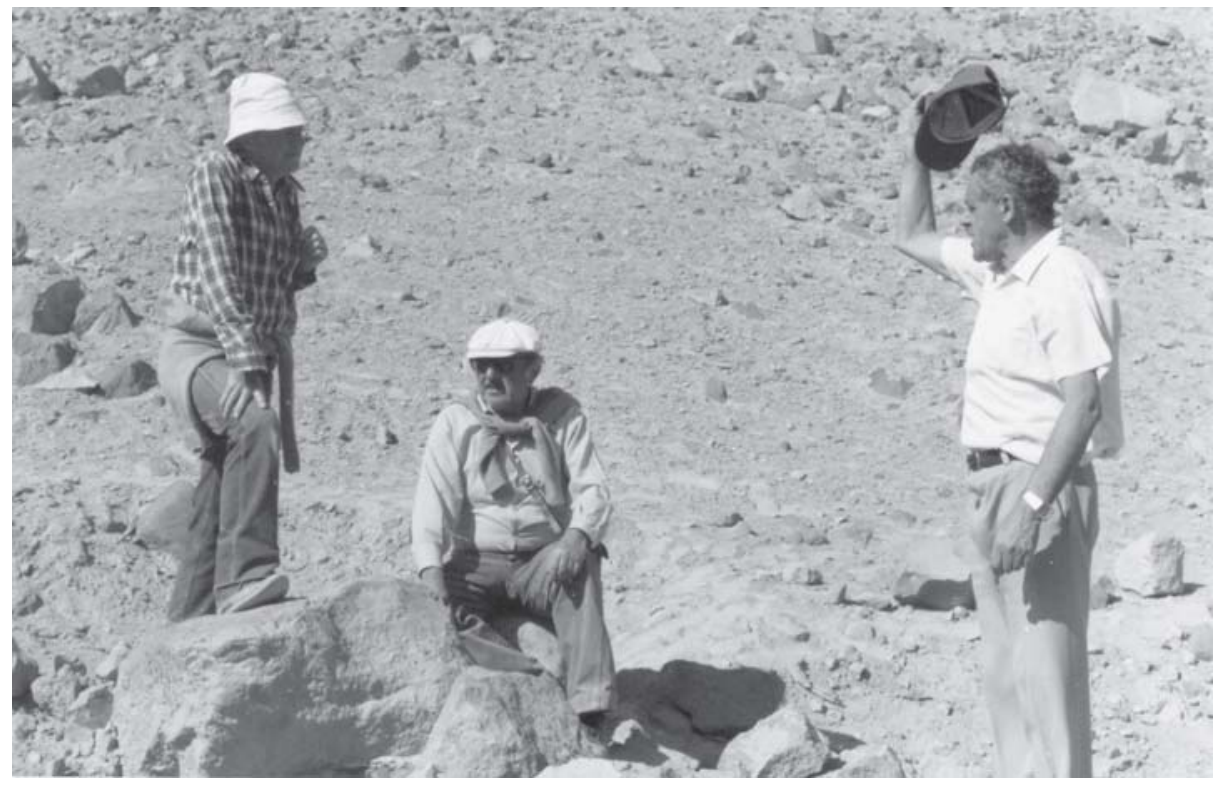

Encuentro de Hans, Virgilio Schiappacasse y Guillermo Focacci en Taltape, valle de Camarones, tres grandes de la arqueología del norte de Chile (invierno de 1983, foto gentileza de C. Santoro). 
intención, la pasión y la fuerza para estar donde estaba el arte rupestre, independiente de las limitaciones del entorno o de su propio cuerpo con el paso de los años. Su compromiso y pasión traspasan las dificultades del relieve en pos de un objetivo mayor: la arqueología y el conocimiento del pasado.

Recuerdo que durante el Taller de la Cultura Aconcagua, celebrado a mediados de los 90, Florence Constantinescu exponía los resultados de una prospección en la alta cordillera andina de Chile central, muy próxima a la frontera y un lugar de difícil acceso. Estaba en ello, describiendo los sitios y su localización, cuando Don Hans la interrumpe para hacerle una pregunta: si ella se refería a un sitio que estaba en un punto $\mathrm{X}$ o Y de tal lugar, pues él recordaba haberlo visto cuando anduvo por esos lugares hacía ya varios años. Esa pregunta fue para mí la contrastación de mi sospecha y creo que es lo que resume la vida de Don Hans: el saber que estemos donde estemos en terreno, sean los lugares más apartados de este país, existe una probabilidad muy alta y cierta que Don Hans ya haya estado ahí hace muchos años haciendo arqueología, cuando no había facilidad de camionetas ni caminos de dos vías ni siquiera caminos pavimentados o electricidad, estaba ahí sólo motivado por el afán del conocer y la pasión por la arqueología. Creo que a través de esa experiencia de vida Don Hans encarna lo que es la Arqueología y hacer Arqueología. Por ello, el mejor homenaje a realizarle pienso que es en ese mismo lugar, el terreno; por un momento detenernos en la vorágine científica de la campaña para saludar a él y sus vivencias, sabiendo que posiblemente hace años o décadas, Don Hans estuvo ahí o en sus cercanías, y que hoy rondan aún por tales espacios las experiencias de sus antiguas campañas, junto a su carpa, una fogata nocturna y conversaciones que construyeron parte de los fundamentos de nuestra prehistoria.

\section{Querido Hans Niemeyer, testimonio de Victoria Castro ${ }^{2}$}

Me he resistido a escribirte desde que se te ocurrió irte de este mundo en la primavera de 2005. Supimos de esta partida lejos, cuando con Varinia Varela, Patricio Núñez, Carlos Aldunate y los alumnos de la U

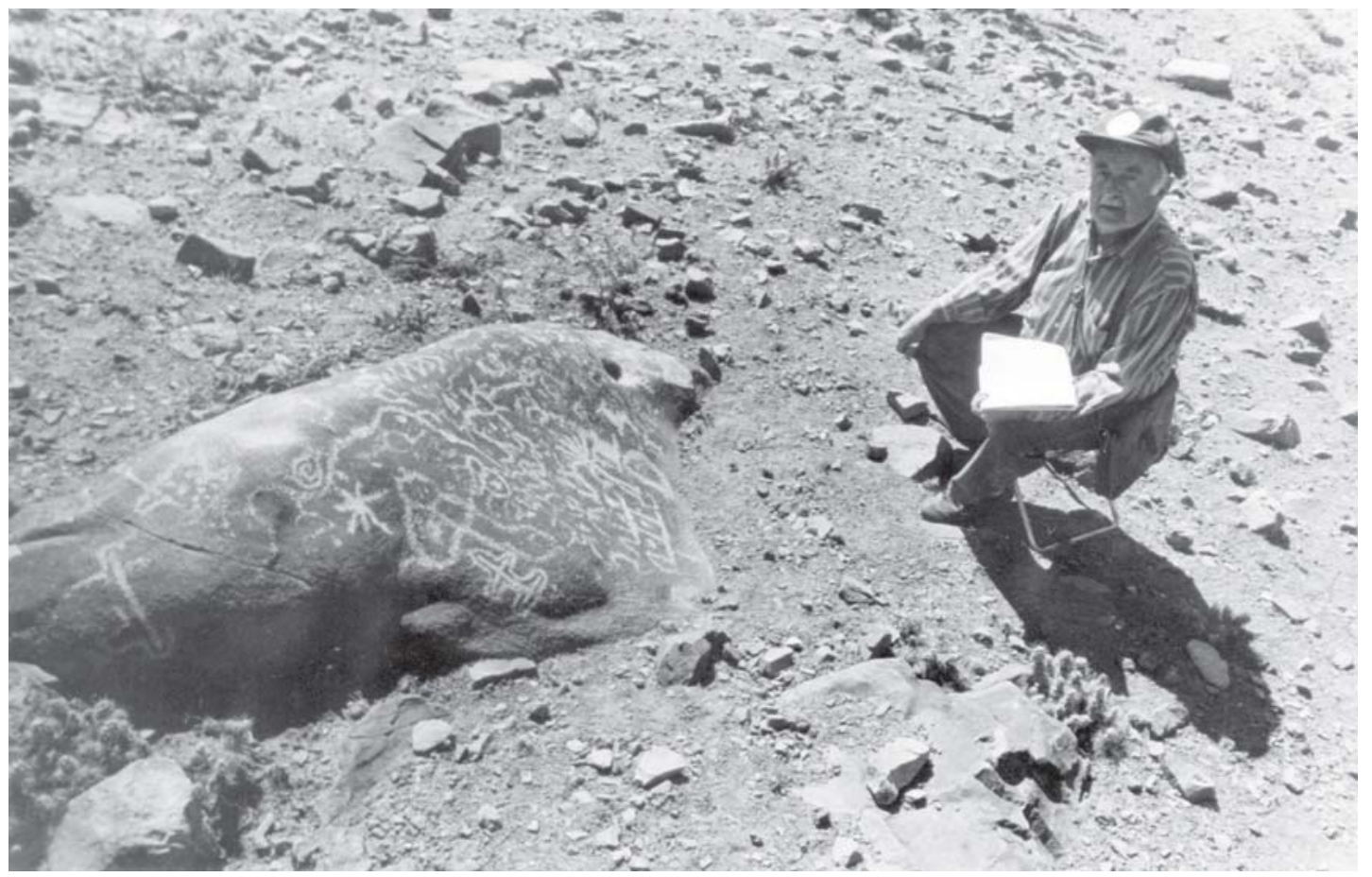

En el valle de Illapel en 1988 (fotografía gentileza de A. Troncoso).

2 Departamento de Antropología, Facultad de Ciencias Sociales, Universidad de Chile. vcastrorojas@hotmail.com 
que nos acompañaban, estábamos en Cobija. Comíamos en ese instante de las delicias del mar Pacífico que tanto amabas; siempre tú caminante entre el mar y la cordillera, señor de estas tierras australes del mundo, señor de la comprensión de la naturaleza, pionero de los paisajes culturales, tú que como nadie supo comprender que razón y sentimiento se encarnaban como conocimiento en la gente. Brindamos por ti con la tristeza de tu partida.

$\mathrm{Al}$ día siguiente te hicimos un sencillo homenaje al redescubrir el petrograbado de Gatico en donde podíamos reconocer la enseñanza de tu análogo de Las Lisas. Que profundidad con la que nos acompañas día a día, si lo difícil es escribirte, porque quisiera volver a verte en todos los planos de la existencia, en todos los intersticios de la vida en que te has quedado prendado y yo prendada de tus enseñanzas.

Un día hace mucho tiempo te sorprendiste hasta las lágrimas (siempre aprecié tu humildad y tu emotividad), cuando recibiste una tarjeta de saludos de fin de año, en donde te nombraba como maestro.

Cómo no hacerlo si tuviste la sensibilidad de enseñarnos con enorme cariño y pasión todo lo que tú aprendiste, siempre alegre, siempre con el detalle de la experiencia, que no necesita de un libro ni de una teoría, porque todo está ahí, en esa completitud de la enseñanza del maestro. En estos días, que remito algunos de los escritos y algunas clases a comentar los temas de sociedad y naturaleza entretejidos, te recuerdo casi cotidianamente.

Probablemente me fasciné primero con tus conferencias, tan vívidas, tan inmensamente generosas. Ya te queríamos, ya estabas instalado en nuestras vidas y, por cierto, en mi familia.

Después vino la formalidad de la formación obligatoria en topografía que tú nos enseñabas. Confieso que la sufrí y la pasé... Pero sobre eso se imponía tu visión de naturalista pleno, con el complemento de tu amigo y compañero Virgilio. Tú nos impregnabas de vuestros quehaceres, con inmensa admiración por el Dr. Schiappacasse. Contigo caminábamos por el valle de Camarones, subíamos a los portezuelos del Norte Chico, nos extendíamos hasta la Patagonia, donde nunca dejaste de alabar estas tierras, porque conociste a tu Selva. Y ella, tu compañera, fue también compañera nuestra en reuniones, congresos y terrenos.

Hans, cómo no recordar tu rigurosidad y seriedad al levantar como nadie la Sociedad Chilena de Arqueología que nos cobija hasta ahora. Tú eres el Cobijo, estás en los inicios de todo, con una actitud de generosidad y humanidad ejemplar incomparable, Hans, alegría de vivir.

Santiago, Invierno 2006.

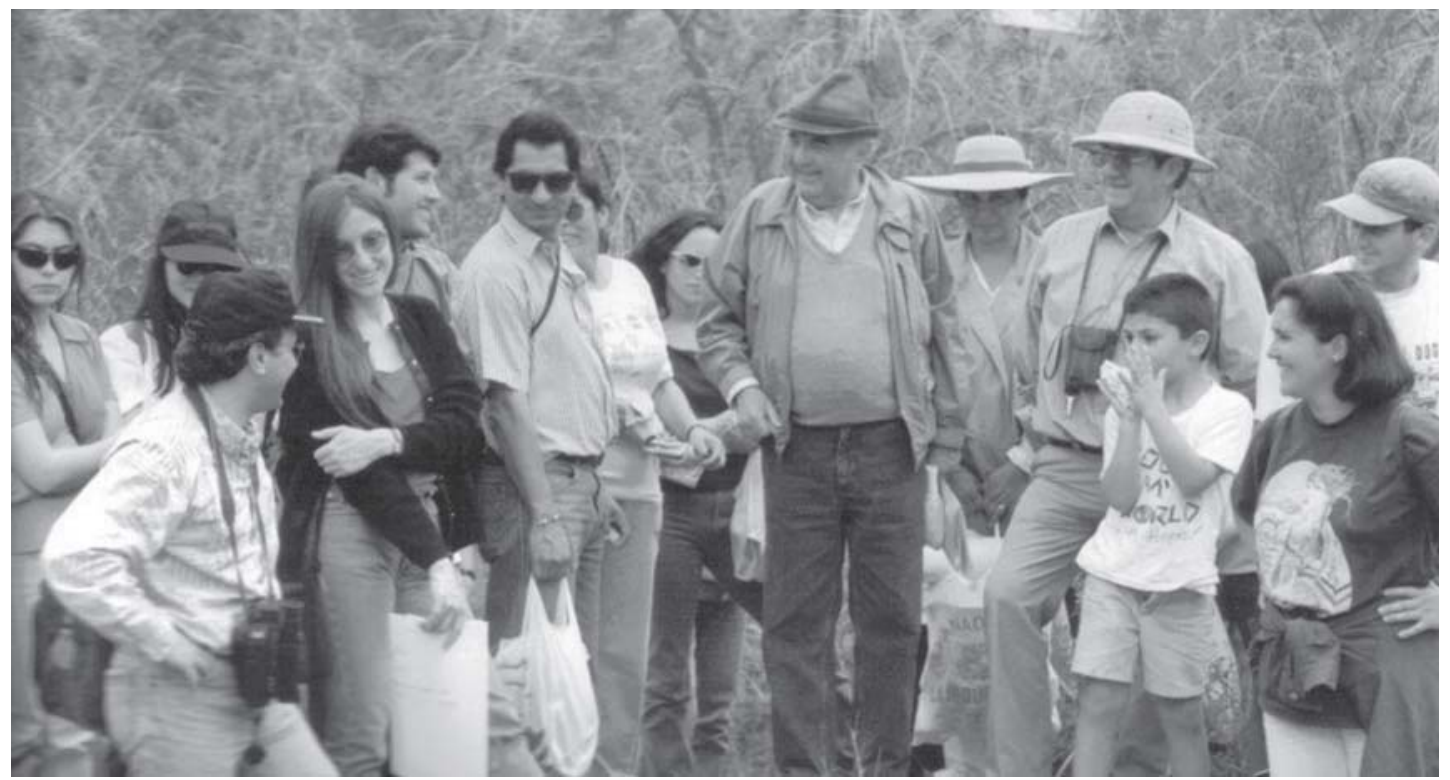

Visita al sitio Iglesia Colorada, después del XIV Congreso Nacional de Arqueología Chilena en Copiapó, octubre 1977 (foto gentileza de A. Troncoso). 


\section{Homenaje al ing. prof. Hans Niemeyer Fernández (1921-2005), testimonio de Lautaro Núñez A. ${ }^{3}$}

El fallecimiento de Hans aunque esperado por su avanzada edad fue sorpresivo puesto que en nuestro imaginario generacional siempre él estaba allí, cumpliendo roles llenos de significado y disciplina que lo involucraron con los inicios científicos de una primera arqueología hecha en Chile. No es fácil descubrir en América Latina una vida entera dedicada a las investigaciones de terreno desde una perspectiva tan intensa que incluyó complejas y verdaderas expediciones a lo largo del país y sus vínculos con las ciencias naturales, hacia el reconocimiento de la diversidad prehistórica del país. Toda su obra está reflejada en la totalidad de los eventos científicos ocurridos en el país desde la década de los cincuenta hasta el presente, incluyendo sus incontables publicaciones que lo distinguieran en nuestro ámbito académico, siempre al servicio de la comunidad científica, exponiéndose tantas veces al debate de sus pares. En un momento en que existían escasísimas publicaciones arqueológicas en Chile, sus artículos con descripciones cuidadosas eran modelos a seguir cuando la docencia antropológica aún no se iniciaba en los claustros universitarios. Marcado, así, por su carácter pionero, sus contribuciones se sucedieron en el tiempo hasta sus últimos momentos, siempre preocupado por publicar todas sus notas provenientes de innumerables viajes sea como contratista, asesor, consultor, académico, en fin, por causas tan diversas que se unían en un objetivo común: explorar, registrar y excavar para su "ciencia adoptada" y esencial.

Para una exhaustiva y futura biografía de Hans he seleccionado algunos testimonios que lo ilustran en materias tan disímiles entre sí. Por el año 1962 me reuní en su particular oficina de la calle Ahumada, que más que un estudio de ingeniería era un laboratorio de arqueología tutelado por su dibujante "privado" y su secretaria-sobrina Hilda Niemeyer. Allí lo sorprendí editando la "Revista Universitaria" de la Academia Chilena de Ciencias Naturales, financiada por la Pontificia Universidad Católica. Me explicaba la importancia de jerarquizar a los grandes naturalistas que aún se mantenían vigentes a través de esa heroica tradición científica representada por Gualterio Looser, Luis Peña, Hugo Gunckel, Rafael Barros, Nibaldo Bahamondes, entre otros. Esto explica que varios anexos de los estudios de Hans provienen de estos especialistas, con quienes compartió una gran amistad al editarles sus aportes con su particular acuciosidad, creando las condiciones absolutamente amables para que los primeros estudios arqueológicos tuvieran en los Anales su órgano casi oficial de difusión, cuando no existía ninguna revista especializada en nuestras disciplinas. Su dedicación a estos emprendimientos editoriales lo destacan hasta el final de sus días y es lejos otro de los atributos más valiosos que se sumaron a su porfiada inclinación hacia una permanente arqueología de campo. Definitivamente, sus manos eran de un arqueólogo genuino, de aquellos que saben caminar, comer, beber, dormir, manejar, cocinar, excavar, publicar y amar, al margen de las ansias desesperadas de poder. Su aporte a la arqueología chilena fue una de las más fecundas en cuanto todo lo que vio lo transformó en sus típicos artículos descriptivos con esa formalidad heredada de sus ancestros y por cierto de la ingeniería que lo llevó a ser quizás el único arqueólogo conocido con mapoteca propia derivada de su pública obsesión por llevar a cabo sus célebres levantamientos topográficos. Por cierto, compartimos un largo terreno a raíz de las excavaciones en Tagua-Tagua, donde me permití acercarme al legendario binomio Niemeyer-Schiappacasse. Sin ellos no podría entenderse el tránsito de la arqueología chilena hacia una disciplina científica. Por fin, interactuamos juntos después de tantos años de amistad y ciencia por "separado". Ahora, entre mastodontes y empanadas "caldúas" tocar esta leyenda viva fue una oportunidad única. A los atributos de Hans, el médico Virgilio puso su arqueología de "quirófano", esto es, el análisis instrumental teórico y metodológico para resolver problemas concretos. Así, mientras Hans se enrabiaba con los alarifes, siguiendo la cota en la cuenca de Tagua-Tagua y Cuchipuy, Virgilio, sobre el piso de faenamiento finipleistocénico, obtenía muestras representativas de sedimentos para identificar microastillamientos de la industria lítica... Ambos tenían la libertad académica para decir

Instituto de Investigaciones Arqueológicas y Museo, Universidad Católica del Norte, San Pedro de Atacama. lautanunez@netline.cl 
en poco tiempo juicios de valor francamente demoledores; ambos cerraban los ojos en los debates como si durmieran para confundir a la audiencia, con observaciones instantáneas y "misilísticas". Ambos hacían gala de una exquisita sensibilidad como el apoyo absoluto que le otorgaron al joven Julio Montané, en sus tiempos heroicos que solo él sabe y recuerda. Ambos se complementaban hasta en las discordias en donde Virgilio solía soltar a su histriónico genovés oculto, mientras que Hans se protegía tras su carácter de panzer prusiano, en veladas memorables arbitradas por la neutralidad de Rodolfo Casamiquela.

Por otra parte, recuerdo aquel día 13 de enero del año 1963 cuando compartíamos por azar la última corrida de asientos entre los 13 participantes del Primer Congreso Nacional y Segundo Internacional de Arqueología, en las dependencias del Museo Arqueológico de San Pedro de Atacama. Había que entregar, nos decía el R.P. Gustavo Le Paige, sugerencias por escrito sobre cómo optimizar estos encuentros, que, en verdad, ya se habían iniciado en Arica, a través del primer Encuentro de Arqueología Internacional, patrocinado por el Museo Regional de Arica. De inmediato le comenté sottovoce que sería importante, siguiendo el modelo de las sociedades científicas de ese entonces, proponer la creación de una Sociedad Chilena de Arqueología: "algo así como la Academia de Ciencias Naturales, con reuniones periódicas y su respectiva publicación..." Fuera de duda, yo estaba impactado por su labor como Editor Científico, desde ya apoyado por Virgilio con quien compartía una vieja amistad... Su respuesta me impresionó por su precisión gestual. Fue un sí con todo el rostro indicándome con la mano que cortara un trozo de papel oficio donde anotaba mis observaciones del evento. Allí escribió la propuesta con nuestros nombres, la que fue leída por Carlos Munizaga y aprobada por unanimidad. Su activa participación en la constitución y consolidación de la Sociedad Chilena de Arqueología fue decisiva para impregnar el destino de lo que llegaría a ser un instrumento de comunicación y perfeccionamiento científico, única en Sudamérica, al mantener hasta hoy su secuencia de 17 Congresos con sus respectivas actas debidamente editadas, desde el año 1961 hasta ahora.

Hans había dispuesto su ideario científico por sobre la vida cotidiana y política y era tan devoto de su quehacer que sentía que nada ni nadie le podría alterar su percepción de "hacer" ciencia, ni siquiera la irracionalidad de una dictadura desatada a la que en principio no le otorgó ninguna trascendencia, a diferencia de sus amigos más cercanos que sabíamos del advenimiento del holocausto. Esto explica que a pocos días del golpe militar se acercara a Antofagasta, convocado por el geólogo Guillermo Chong, quien acababa de descubrir las pinturas del Médano. Guillermo había preparado una expedición entre los tres... Ambos entendieron mi excusa, puesto que el control militar hacia el sur nos podría complicar el paso en momentos en que las radios anunciaban las así llamadas "listas negras". Hans y Guillermo lo hicieron, volviendo en estado de encantamiento. A los años me incorporé a este estudio de Hans. Había prevalecido de nuevo su imperiosa y urgente necesidad de reconocer esas maravillas de la quebrada del Médano. Al respecto, se ha comentado que Hans favoreció a varios amigos comunes que de uno u otro modo se vieron involucrados con las persecuciones y martirios de los primeros años de la dictadura. Allí, en el Médano comentamos este tema y su respuesta lacónica lo refleja en esa acción propia de su conducta: “¿y qué otra cosa podía hacer?” Es cierto que solía emprender acciones inesperadas como preparar una expedición bajo un estado de guerra. Pero ya algo antes del golpe militar estuvo en el centro de otra performance insólita. Resulta que el gerente de la Editorial Quimantú, el senador Chelén, se había interesado en publicar una obra de arqueología que mostrara el pasado indígena en el desierto tarapaqueño para contrastarlo con lo conocido en la Puna de Atacama. Fue así que nuestro manuscrito "El Valle de Tarapacá: 10 Mil Años de Historia" ingresó a talleres de la más temida Editorial Quimantú de la Unidad Popular. Por cierto, Hans lo sabía, y en un acto casi suicida ingresó allí cuando se sacaba a una pira monumental, en carretillas, todo lo que tanto había irritado a la oposición cívico-militar a lo largo de todos los quioscos de Chile... Su descripción del cómo logró sustraer ese manuscrito en su eterno maletín de cuero, entre saludos respetables y prisioneros con las manos en alto, lo demuestra en el perfil de un "justiciero", toda vez que había una edición por medio a punto de ser eliminada. Eran esos gestos casi insensatos que lo cubrieron de fama y aventura en reiteradas oportunidades de su vida, tantas que Selva, su definitivo alter ego y compañera total, podrá recordar estas riesgosas y ahora sabrosas acciones de Hans. Claro, esta obra será edita- 
da como si fuera un "Moisés" salvado de las llamas, con un homenaje al amigo inolvidable. Hans siempre vivió abrazado a la inseparable "enredadera" de "su" Selva, puesto que sin ella se habría perdido para siempre sin ese rumbo norte necesario, con riesgo a desnivelarse sin altura de mira... En este largo peregrinar su taquímetro mantiene hasta ahora los secretos que caen con él, a plomo, hacia el mismo centro de la tierra.

Selva y Hans, su hijo Geólogo por excelencia, su hija, y tantos descendientes y amigos comunes, tendremos el tiempo para recordarlo siempre vivo, apegado a las memorias, porque solo así quien nos dio tanto tiene el derecho a no ser olvidado jamás... 
